\title{
Desempenho dos municípios da região metropolitana do Rio de Janeiro nas hospitalizações por Covid-19. Estudo baseado no SIVEP-Gripe
}

Performance of cities in the metropolitan region of Rio de Janeiro in hospitalizations for Covid-19. SIVEP-Gripe based study

Desempeño de los municipios de la región metropolitana de Río de Janeiro en hospitalizaciones por Covid-19. Estudio basado en SIVEP-Gripe

E-mail: daysecorreia@id.uff.br

Arthur Willkomm Kazniakowski

ORCID: https://orcid.org/0000-0003-0187-2751 Universidade Federal Fluminense, Brasil

E-mail: arthurwillkomm@id.uff.br

Beatriz Nayra Dias de Andrade

ORCID: https://orcid.org/0000-0003-2007-2741 Universidade Federal Fluminense, Brasil

E-mail: bedias@id.uff.br Cínthia Melo Arêas

ORCID: https://orcid.org/0000-0002-1805-7852 Universidade Federal Fluminense, Brasil E-mail: cinthiaareas@id.uff.br Jessyca Martins Lima da Silva

ORCID: https://orcid.org/0000-0003-4480-8100 Universidade Federal Fluminense, Brasil

E-mail: jessycamartins@id.uff.br Juliana Vianna de Freitas

ORCID: https://orcid.org/0000-0002-3389-6697 Universidade Federal Fluminense, Brasil E-mail: julianaviannafreitas@id.uff.br

Lara Toretta Campo Dall'Orto

ORCID: https://orcid.org/0000-0001-6562-7267 Universidade Federal Fluminense,Brasil E-mail: laracampo@id.uff.br. Larissa dos Santos Sebould Marinho.

ORCID: https://orcid.org/0000-0002-5447-0847 Universidade Federal Fluminense,Brasil

E-mail: larissasebould@id.uff.br

Marina Soares de Almeida e Silva

ORCID: https://orcid.org/0000-0002-3664-5164 Universidade Federal Fluminense, Brasil

E-mail: marinaalmeida@id.uff.br

Melissa Corrêa Leal Gardengui

ORCID: https://orcid.org/0000-0001-9046-464X Universidade Federal Fluminense, Brasil E-mail: melissagardengui@id.uff.br 


\author{
Micael Paes Lessa \\ ORCID: https://orcid.org/0000-0003-2379-7190 \\ Universidade Federal Fluminense, Brasil \\ E-mail: mlgarciarosa@id.uff.br \\ Sarah Melo da Costa \\ ORCID: https://orcid.org/0000-0002-3204-8848 \\ Universidade Federal Fluminense, Brasil \\ E-mail: sarahmelo@id.uff.br \\ Sarah Pientznauer Ozório Costa \\ ORCID: https://orcid.org/0000-0003-0029-2202 \\ Universidade Federal Fluminense, Brasil \\ E-mail: sarahpientznauer@id.uff.br \\ Maria Luiza Garcia Rosa \\ ORCID: https://orcid.org/0000-0002-4508-256X \\ Universidade Federal Fluminense, Brasil \\ E-mail: mlgarciarosa@id.uff.br
}

\title{
Resumo
}

Introdução: a preocupação quanto à capacidade de atendimento em saúde, principalmente hospitalar, aumenta com o recrudescimento da epidemia do Covid-19. Objetivo: analisar dados do SIVEP-Gripe de 18 municípios da região metropolitana do Rio de Janeiro, de pacientes internados por SRAG em hospitais públicos e privados. Métodos: é um estudo epidemiológico ecológico e de coorte histórica com o município e o paciente como unidades de análise, no período de março a novembro de 2020. Foram estimadas as razões de chances para sinais e sintomas, fatores de risco, se hospital público ou privado, internação em UTI e óbitos. Empregou-se o teste do qui-quadrado de Pearson $(p<0,05)$. Resultados: somente Niterói internou $>90 \%$ dos seus residentes. Pelo menos $25 \%$ dos hospitais, públicos e privados, internaram somente um paciente levando à dificuldade de aprendizado. Os hospitais públicos apresentaram maior chance de terem internado pacientes mais velhos, de cor de pele preta ou parda, com dispneia, desconforto respiratório e baixa saturação de oxigênio, doença hepática e imunossupressão, condições de maior risco para resultados negativos. A chance de um paciente ter passado por uma UTI em um hospital público foi cerca da metade do hospital privado e a de morte foi cerca do triplo. Conclusão: os municípios estudados, exceto Niterói, não estão preparados para dar suporte a seus residentes na pandemia de Covid-19. Verificou-se discrepância no atendimento dos hospitais públicos e privados, assim como falhas na atenção básica levando a um maior risco de resultados desfavoráveis e um elevado número de óbitos nas unidades hospitalares públicas.

Palavras-chave: Síndrome respiratória aguda grave; Covid-19; Hospitalização; Unidade de terapia intensiva; Óbito.

\begin{abstract}
Introduction: the concern about the capacity of health care, mainly hospital, increases with the upsurge of the Covid19 epidemic. Objective: to analyze SIVEP-Gripe data from 18 municipalities in the metropolitan region of Rio de Janeiro, of patients hospitalized for SARS in public and private hospitals. Methods: It is an ecological epidemiological study and a historical cohort with the cities and patients as units of analysis, from March to November 2020. Odds ratios were estimated for signs and symptoms, risk factors, whether public or private hospital, ICU admissions and deaths. Pearson's chi-square test $(\mathrm{p}<0,05)$ was used. Results: Only Niterói admitted $>90 \%$ of its residents. At least $25 \%$ of hospitals, public and private, admitted only one patient, leading to learning difficulties. Public hospitals were more likely to have older patients, black or brown skin color, with dyspnea, respiratory distress and low oxygen saturation, liver disease and immunosuppression, conditions that are at greater risk for negative outcomes. The chance of a patient having been in an ICU in a public hospital is about half of the private hospital and the chance of death was about three times. These results indicate that patients admitted to public hospitals had a failed assistance from primary care, since they had more signs and symptoms pointing to a negative outcome, indicating a delay in hospitalization. Conclusion: the studied municipalities, except Niterói, are not prepared to support their residents in the Covid-19 pandemic. There was a discrepancy in the attendance of public and private hospitals, as well as failures in primary care leading to a greater risk of unfavorable results and a high number of deaths in public hospital units.
\end{abstract}

Keywords: Severe acute respiratory syndrome; Covid-19; Hospitalization; Intensive care unit; Deaths.

\section{Resumen}

Introducción: la preocupación debido a la capacidad del atendimiento de la salud, especialmente en los hospitales, aumenta con el agravamiento de la epidemia del Covid-19. Objetivo: analizar los datos del SIVEP-Gripe de 18 municipios de la región metropolitana de Río de Janeiro, de pacientes hospitalizados por SARS en hospitales públicos y privados. Métodos: es un estudio epidemiológico ecológico y de cohorte histórico con el municipio y el paciente como unidades de análisis, de marzo a noviembre de 2020. Se estimaron las razones de probabilidad para las marcas y 
síntomas, los factores de riesgo, ya sea en hospitales públicos o privados, en la UCI y óbito . Se utilizó la prueba de chi-cuadrado de Pearson ( $\mathrm{p}<0,05$ ). Resultados: solo Niterói admitió > 90\% de sus residentes. Al menos el 25\% de los hospitales, públicos y privados, admitieron solo un paciente, lo que provocó dificultades de aprendizaje. Los hospitales públicos tenían más probabilidades de hospitalizar pacientes mayores, negros o morenos, con disnea, dificultad respiratoria y baja saturación de oxígeno, enfermedad hepática e inmunosupresión, condiciones de mayor riesgo de resultados negativos. La probabilidad de que un paciente haya estado en una UCI de un hospital público es aproximadamente la mitad de la del hospital privado y la probabilidad de muerte es aproximadamente tres veces mayor. Estos resultados indican que los pacientes ingresados en hospitales públicos tuvieron asistencia de atención primaria precaria ya que tenían más marcas y síntomas que apuntaban a un resultado negativo, lo que indica un retraso en la hospitalización. Conclusión: los municipios estudiados, excepto Niterói, no están preparados para apoyar a sus residentes en la pandemia Covid-19. Hubo una discrepancia en la asistencia de los hospitales públicos y privados, así como fallas en la atención primaria que llevaron a un mayor riesgo de resultados desfavorables y un alto número de muertes en unidades hospitalarias públicas.

Palabras clave: Síndrome respiratorio agudo severo; Covid-19; Hospitalización; Unidad de cuidados intensivos; Óbito.

\section{Introdução}

A COVID-19 se disseminou de forma desigual na Região Metropolitana do Rio de Janeiro até final de julho de 2020. Conforme dados publicados recentemente, com informações de 18 municípios da Região Metropolitana do Rio de Janeiro (RM), observou-se que municípios menos vulneráveis apresentaram maior incidência e os gráficos de casos mostraram uma tendência à estabilidade na maior parte dos municípios até o final da observação (Kawa et al, 2020).

E a partir do início do mês de novembro passou ocorrer no Brasil o que aconteceu um pouco antes nos Estados Unidos e na Europa, vivenciando o que se chamou de $2^{\mathrm{a}}$ segunda onda (Fiocruz, 2020). Ainda, é preciso esclarecer que o padrão de ocorrência de casos no nosso país é bem diferente do que acontece na Europa, onde na maioria dos países o número de casos diminuiu muito, com índice de reprodução R muito inferiores a 1, o que não foi o caso do Brasil (Johns Hopkins University of Medicine, 2020).

Particularmente no Estado do Rio de Janeiro, a imprensa vem noticiando um aumento do número de casos. Por exemplo, no dia 20/12 a média móvel de mortes estava em alta pelo sexto dia consecutivo no estado, segundo consórcio dos veículos de imprensa que consolida informações enviadas pelas Secretarias Estaduais de Saúde (G1 Portal de notícias da Globo, 2020). E que, distintos níveis de atenção nas unidades territoriais estariam operando além da sua capacidade, comprometendo a atenção de pacientes principalmente aqueles com maior gravidade, apontando para uma situação crítica do sistema no atendimento desta demanda (Noronha et al, 2020).

O Sistema Único de Saúde, o SUS, um sistema de saúde universal, foi fundamental na diminuição dos danos da pandemia no país. O fortalecimento da regionalização do SUS, nos mais diferentes níveis de atenção teria sido essencial para diminuirmos os danos à população, com o auxílio da criação de redes de saúde intermunicipais. Visto que, a diferença "hierárquica" da disponibilidade de tecnologias visa maior integralidade do cuidado. E que, frente ao contexto epidemiológico e da evolução da pandemia, a expansão e qualificação profissional especializada desde a atenção básica, aumento da disponibilização de serviços hospitalares e melhora do sistema de logística e da gestão do sistema, teriam sido de suma importância (Frente pela vida, 2020).

É importante lembrar que, desde 2000, existe o Consórcio Intermunicipal de Saúde da Baixada Fluminense CISBAF. Conta com 11 municípios dessa região e apresenta como principal objetivo, o combate aos problemas comuns relacionados à saúde de maneira conjunta, mais efetiva e, devido a união, mais favorecida financeiramente falando (CISBAF, 2020). 
A preocupação quanto ao atendimento dos serviços de saúde, especificamente dos hospitais, no cuidado adequado aos pacientes durante essa segunda onda também alcançou outros países, como por exemplo, os Estados Unidos (Goldberg, 2020; Karac-Mandic 2020).

Diante desse cenário de aumento de número de casos e sobrecarga dos serviços de saúde, o objetivo do presente estudo foi analisar dados do SIVEP-Gripe de 18 municípios da região metropolitana do Rio de Janeiro, de pacientes internados por SRAG em hospitais públicos e privados.

\section{Metodologia}

Trata-se de um estudo ecológico e de coorte histórica, quantitativo, utilizando-se duas unidades de análise, respectivamente: o município e o paciente, ambos considerando informações a partir de dados secundários, do período de março a novembro de 2020. Utilizou-se a metodologia de Análise de Situação de Saúde (ASIS) conforme proposto pelo Ministério da Saúde (Brasil, 2015).

A região metropolitana do Estado do Rio de Janeiro em sua concepção geoeconômica (RMG), inclui 18 municípios localizados ao redor da capital fluminense: os 13 municípios da Baixada Fluminense (Itaguaí, Seropédica, Paracambi, Japeri, Queimados, Nova Iguaçu, Mesquita, Nilópolis, Belford Roxo, São João de Meriti, Duque de Caxias, Magé e Guapimirim) e os 5 municípios do Leste Metropolitano (Niterói, São Gonçalo, Itaboraí, Maricá e Tanguá) (IPEA, 2020).

Foram utilizados dados de hospitalizações por COVID-19 das notificações por SRAG (Síndrome respiratória aguda grave) obtidas no Sistema de Informação de Vigilância da Gripe (SIVEP-Gripe) (Brasil, 2020 1) inseridos no sistema até 07/12/2020.

A vigilância da SRAG no Brasil é realizada pelo Ministério da Saúde desde 2009, a partir da pandemia de Influenza A (H1N1). Em 2020 a vigilância da COVID-19 foi incorporada a este monitoramento (Brasil, 2020).

É considerado caso de SRAG (SRAG-hospitalizado), o indivíduo hospitalizado com febre, mesmo que referida, acompanhada de tosse ou dor de garganta e que apresente dispneia ou saturação de $\mathrm{O} 2<95 \%$ ou desconforto respiratório ou que evoluiu para óbito por SRAG independente de internação (Brasil, 2020). Em 2020 observou-se que mais de 97\% dos casos de SRAG eram de COVID-19. (Agencia Brasil, 2020) A população foi estimada para cada município em 2019 e 2020, segundo o IBGE (IBGE, 2019; 2020).

As informações referentes ao regime dos hospitais (público ou privado), à disponibilidade de leitos hospitalares, UTIs e às hospitalizações foram obtidas no banco de dados de Síndrome Respiratória Aguda Grave (Brasil, 2020 1) e no Cadastro Nacional de estabelecimentos de Saúde (Brasil, 2020).

\section{Análise estatística}

As características dos municípios e dos pacientes foram apresentadas como frequência absoluta e relativa, e concentração de leitos hospitalares gerais e complementares (UTI, entre outros) em leito por 100 mil habitantes (Tabelas 1 e 2). O número total de hospitais, públicos e privados, que internaram os pacientes analisados foi apresentado em frequência absoluta e o número de internações, em números absolutos e quartis (Tabela 3). Foram estimadas as razões de chances e seus intervalos de confiança de $95 \%$ de haver sinais e sintomas e fatores de risco (Tabelas $4.1 \mathrm{e} 4.2$ ). E igualmente foram estimadas razões de chance e seus intervalos de confiança de $95 \%$ por regime do hospital (público ou privado) para ter sido internado em UTI e evoluído para óbito (Tabela 5). Em ambos os casos as diferenças foram testadas com o teste do qui-quadrado de Pearson, com correção de continuidade de Yates. A significância estatística ficou estabelecida em $p<0,05$. O pacote estatístico utilizado na análise foi o Statistical Package for the Social Sciences (SPSS) 21 (IBM Corp. Released, 2012). 


\section{Aspectos Éticos}

Conforme os preceitos estabelecidos na Resolução 510/2016, tal tipo de pesquisa não necessitou de avaliação do sistema CEP/CONEP.

\section{Resultados}

As características demográficas dos municípios e dos pacientes internados por SRAG e fatores de risco estão na Tabela 1. Tanto a população quanto a densidade demográfica variaram muito entre os municípios. Além disso, observou-se grande variação quanto ao percentual de homens, idosos, indivíduos com cor da pele preta ou parda. E que somente quatro entre os 18 municípios tiveram menos de 50\% dos internados com SRAG com fatores de risco.

Tabela 1. Características demográficas dos 18 municípios (A e B) da região metropolitana do Estado do Rio de Janeiro e dos pacientes internados por SRAG, de janeiro a novembro de 2020 (C a F). Niterói,2020.

\begin{tabular}{|c|c|c|c|c|c|c|}
\hline $\begin{array}{l}\text { Município de } \\
\text { residência }\end{array}$ & $\begin{array}{c}\text { A } \\
\text { População } \\
\mathrm{n} \\
\end{array}$ & $\begin{array}{c}\text { B } \\
\text { Densidade } \\
\text { demográfica } \\
\text { hab } / \mathrm{km}^{2} \\
\end{array}$ & $\begin{array}{c}\mathrm{C} \\
\text { Homens } \\
\mathrm{n}(\%) \\
\end{array}$ & $\begin{array}{c}\mathrm{D} \\
\text { Idade }>65 \\
\text { anos } \\
\mathrm{n}(\%) \\
\end{array}$ & $\begin{array}{c}\mathrm{E} \\
\text { Cor da pele (preta } \\
\text { ou parda) } \\
\mathrm{n}(\%)\end{array}$ & $\begin{array}{c}\mathrm{F} \\
\text { Fator de risco } \\
\mathrm{n}(\%)\end{array}$ \\
\hline Belford Roxo & 510906 & 6031,38 & $38(55,1)$ & $30(43,5)$ & $27(39,1)$ & $43(62,3)$ \\
\hline Duque de Caxias & 919596 & 1828,51 & $127(49,2)$ & $113(43,8)$ & $92(35,7)$ & $163(63,2)$ \\
\hline Guapimirim & 60517 & 142,70 & $11(42,3)$ & $16(61,5)$ & $13(50,0)$ & $22(84,6)$ \\
\hline Itaboraí & 240592 & 506,55 & $44(64,7)$ & $34(50,0)$ & $13(19,1)$ & $41(60,3)$ \\
\hline Itaguaí & 133019 & 395,45 & $20(48,8)$ & $24(58,5)$ & $15(36,6)$ & $26(63,4)$ \\
\hline Japeri & 104768 & 1166,37 & $9(56,3)$ & $4(25,0)$ & $7(43,8)$ & $6(37,5)$ \\
\hline Magé & 245071 & 585,13 & $34(53,1)$ & $33(51,6)$ & $17(26,6)$ & $31(48,4)$ \\
\hline Maricá & 161207 & 351,55 & $26(59,1)$ & $26(59,1)$ & $21(47,7)$ & $23(52,3)$ \\
\hline Mesquita & 176103 & 4310,48 & $25(50,0)$ & $30(60,0)$ & $23(46,0)$ & $28(56,0)$ \\
\hline Nilópolis & 162485 & 8117,62 & $27(48,2)$ & $32(57,1)$ & $19(33,9)$ & $35(62,5)$ \\
\hline Niterói & 513584 & 3640,80 & $167(50,2)$ & $169(50,8)$ & $88(26,4)$ & $219(65,8)$ \\
\hline Nova Iguaçu & 821128 & 1527,60 & $116(54,5)$ & $113(53,1)$ & $71(33,3)$ & $121(56,8)$ \\
\hline Paracambi & 52257 & 262,27 & $5(55,6)$ & $5(55,6)$ & $6(66,7)$ & $3(33,3)$ \\
\hline Queimados & 150319 & 1822,60 & $13(65,0)$ & $12(60,0)$ & $7(35,0)$ & $12(60,0)$ \\
\hline São Gonçalo & 1084839 & 4035,90 & $175(47,8)$ & $170(46,4)$ & $141(38,5)$ & $205(56,0)$ \\
\hline São João de Meriti & 472406 & 13024,56 & $62(48,8)$ & $53(41,7)$ & $39(30,7)$ & $72(56,7)$ \\
\hline Seropédica & 82312 & 275,53 & $8(61,5)$ & $6(46,2)$ & $10(76,9)$ & $4(30,8)$ \\
\hline Tanguá & 34309 & 211,21 & $9(64,3)$ & $5(35,7)$ & $6(42,9)$ & $9(64,3)$ \\
\hline
\end{tabular}

*hab: habitantes

Fonte: https://www.saude.rj.gov.br/informacao-sus/fichas-tecnicas-municipais/ https://opendatasus.saude.gov.br/dataset/bd-srag-2020.

Os municípios de São Gonçalo, Niterói, Duque de Caxias e Nova Iguaçu foram os que mais internaram pacientes com SRAG, e são também os mais populosos (Tabela 1), mas não são os municípios com maior número de leitos totais ou complementares (UTI) da região. Niterói é o município com maior concentração de leitos totais e complementares da região. Chama atenção a situação de Tanguá e Guapimirim, dois municípios pequenos, com alta concentração de leitos totais, mas que não possuem nenhum leito complementar.

Na Tabela 2, observa-se que Niterói foi o único dos 18 municípios que internou a quase totalidade de seus residentes (93,1\%). 
Tabela 2. Disponibilidade de leitos e hospitalizações por SRAG em 18 municípios da região metropolitana do estado do Rio de Janeiro, de janeiro a novembro de 2020. Niterói,2020.

\begin{tabular}{|c|c|c|c|c|c|c|c|}
\hline $\begin{array}{l}\text { Município de } \\
\text { residência }\end{array}$ & $\begin{array}{c}\text { Total de } \\
\text { internações }\end{array}$ & Leitos Totais** & $\begin{array}{c}\text { Leitos } \\
\text { Complementares*** }\end{array}$ & $\begin{array}{c}\text { Internações } \\
\text { no } \\
\text { município } \\
\text { de } \\
\text { residência }\end{array}$ & $\begin{array}{l}\text { Internação fora } \\
\text { do município } \\
\text { de residência }\end{array}$ & UTI & Óbito \\
\hline & $\mathrm{n}(\%)^{*}$ & $\begin{array}{l}\text { por } 100 \mathrm{mil} \\
\text { habitantes }\end{array}$ & por 100 mil habitantes & $\mathrm{n}(\%)$ & $\mathrm{n}(\%)$ & $\mathrm{n}(\%)$ & $\mathrm{n}(\%)$ \\
\hline Belford Roxo & $69(3.9)$ & 82.79 & 22.12 & $7(10,8)$ & $\begin{array}{l}\text { Rio de Janeiro } \\
\quad 37(56,9)\end{array}$ & $25(36,2)$ & $17(24,6)$ \\
\hline $\begin{array}{l}\text { Duque de } \\
\text { Caxias }\end{array}$ & $258(14.4)$ & 121.36 & 69.38 & $140(57,4)$ & $\begin{array}{l}\text { Rio de Janeiro } \\
\quad 86(35,2)\end{array}$ & $98(38,0)$ & $83(32,2)$ \\
\hline Guapimirim & $26(1.5)$ & 228.04 & 0.00 & $19(73,1)$ & $\begin{array}{l}\text { Rio de Janeiro } \\
\quad 4(15,4)\end{array}$ & $13(50,0)$ & $9(34,6)$ \\
\hline Itaboraí & $68(3.8)$ & 135.50 & 21.20 & $32(50)$ & $\begin{array}{c}\text { Niterói } 10 \\
(15,6)\end{array}$ & $22(32,4)$ & $22(32,4)$ \\
\hline Itaguaí & $41(2.3)$ & 130.06 & 11.28 & $29(74,4)$ & $\begin{array}{l}\text { Rio de Janeiro } \\
10(25,6)\end{array}$ & $8(19,5)$ & $14(34,1)$ \\
\hline Japeri & $16(0.9)$ & 19.09 & 8.59 & $8(50)$ & $\begin{array}{c}\text { Rio de Janeiro } \\
3(18,8)\end{array}$ & $4(25,0)$ & $2(12,5)$ \\
\hline Magé & $64(3.6)$ & 67.74 & 13.47 & $23(35,9)$ & $\begin{array}{c}\text { Petrópolis } \\
15(23,4)\end{array}$ & $20(31,3)$ & $29(45,3)$ \\
\hline Maricá & $44(2.5)$ & 165.63 & 27.91 & $22(50)$ & $\begin{array}{c}\text { Niterói } \\
14(31,8)\end{array}$ & $21(47,7)$ & $14(31,8)$ \\
\hline Mesquita & $50(2.8)$ & 102.21 & 14.20 & $10(21,7)$ & $\begin{array}{l}\text { Rio de Janeiro } \\
\quad 24(52,2)\end{array}$ & $19(38,0)$ & $15(30,0)$ \\
\hline Nilópolis & $56(3.1)$ & 152.01 & 63.39 & $11(21,2)$ & $\begin{array}{l}\text { Rio de Janeiro } \\
36(69,2)\end{array}$ & $17(30,4)$ & $19(33,9)$ \\
\hline Niterói & 333 (18.6) & 405.97 & 87.62 & $295(93,1)$ & $\begin{array}{c}\text { Rio de Janeiro } \\
15(4,7)\end{array}$ & $116(34,8)$ & $51(15,3)$ \\
\hline Nova Iguaçu & 213 (11.9) & 112.28 & 29.96 & $79(79,9)$ & $\begin{array}{l}\text { Rio de Janeiro } \\
\quad 89(44,9)\end{array}$ & $80(37,6)$ & $60(28,2)$ \\
\hline Paracambi & $9(0.5)$ & 185.62 & 15.31 & $8(88,9)$ & $\begin{array}{l}\text { Rio de Janeiro } \\
1(11,1)\end{array}$ & $2(22,2)$ & $3(33,3)$ \\
\hline Queimados & $20(1.1)$ & 79.16 & 6.65 & $0(0,00)$ & $\begin{array}{c}\text { Rio de Janeiro } \\
15(78,9)\end{array}$ & $8(40,0)$ & $5(25,0)$ \\
\hline São Gonçalo & $366(20.5)$ & 144.54 & 35.58 & $224(64,2)$ & $\begin{array}{l}\text { Niterói } 100 \\
(28,7)\end{array}$ & $103(28,1)$ & $76(20,8)$ \\
\hline $\begin{array}{l}\text { São João de } \\
\text { Meriti }\end{array}$ & $127(7.1)$ & 29.42 & 26.04 & $22(21)$ & $\begin{array}{l}\text { Rio de Janeiro } \\
\quad 61(58,1)\end{array}$ & $48(37,8)$ & $39(30,7)$ \\
\hline Seropédica & $13(0.7)$ & 43.74 & 6.07 & $1(10)$ & $\begin{array}{l}\text { Rio de Janeiro } \\
6(60)\end{array}$ & $4(30,8)$ & $5(38,5)$ \\
\hline Tanguá & $14(0.8)$ & 623.74 & 0.00 & $5(41,7)$ & $\begin{array}{l}\text { Rio Bonito } 6 \\
(50)\end{array}$ & $2(14,3)$ & $5(35,7)$ \\
\hline
\end{tabular}

*(entre municípios)

$* *$ (menos complementares)

***(UTI pediátrico e adulto)

Fonte: https://opendatasus.saude.gov.br/dataset/bd-srag-2020 e http://cnes.datasus.gov.br.

E que treze dos dezoito municípios da região internaram menos de $70 \%$ dos seus residentes. O município do Rio de Janeiro internou mais de $30 \%$ de onze dos dezoito municípios da região. A prevalência de internação em UTI variou 14,3\% a $50 \%$ e o percentual de óbitos variou de $12,5 \%$ a $45,3 \%$ (Tabela 2 ).

Foram internados, no período analisado, 1679 residentes nos 18 municípios da RM, em 205 unidades hospitalares, a maioria pública $(>63 \%)$. Entre os hospitais públicos, $75 \%$ das internações ocorreram em unidades que internaram menos de 8 pacientes por SRAG. Nos hospitais privados o $3^{\circ}$ quartil correspondeu a 9 internações. Ressalta-se que pelo menos $25 \%$ dos hospitais, públicos e privados, internaram somente um paciente (Tabela 3). 
Tabela 3. Hospitais públicos e privados que internaram residentes dos 18 municípios da Região Metropolitana do Rio de Janeiro por SRAG, de março a novembro de 2020.Niterói,2020.

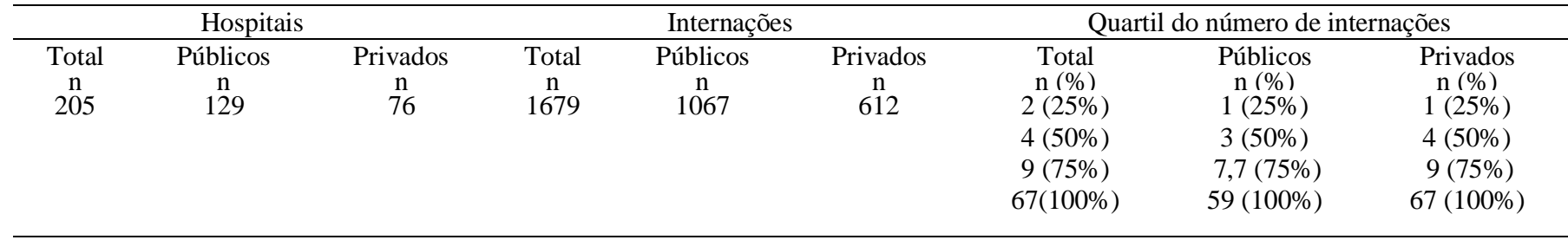

Fonte: https://opendatasus.saude.gov.br/dataset/bd-srag-2020.

As Tabelas 4.1 e 4.2 exibem as razões de chances de apresentar sinais e sintomas ou fatores de risco se internados em hospitais públicos ou privados. Ou seja, dispneia, desconforto respiratório e baixa saturação de oxigênio <95\% tiveram significância estatística (pvalor < 0,05), havendo mais pessoas internadas com esses sinais ou sintomas nos hospitais públicos do que nos hospitais privados (Tabela 4.1).

Tabela 4.1. Razões de Chances para sinais ou sintomas entre pacientes internados em hospitais públicos em relação aos hospitais privados em 18 municípios da Região Metropolitana do estado do Rio de Janeiro entre março e novembro de 2020. Niterói,2020.

\begin{tabular}{|c|c|c|c|c|}
\hline & $\begin{array}{c}\text { Hospitais } \\
\text { públicos (n) }\end{array}$ & $\begin{array}{c}\text { Hospitais } \\
\text { privados (n) }\end{array}$ & OR (IC 95\%) & $\mathrm{P}$ valor* \\
\hline \multicolumn{5}{|l|}{ Sinais ou sintomas } \\
\hline Tosse & & & $0,758(0,577-1,031)$ & 0,090 \\
\hline Sim & 618 & 431 & & \\
\hline Não & 140 & 74 & & \\
\hline Febre & & & $0,779(0,590-1,029)$ & 0,091 \\
\hline Sim & 572 & 400 & & \\
\hline Não & 178 & 97 & & \\
\hline Dor de garganta & & & $0,744(0,531-1,044)$ & 0,104 \\
\hline Sim & 95 & 81 & & \\
\hline Não & 375 & 238 & & \\
\hline Dispneia & & & $1,705(1,286-2,261)$ & 0,000 \\
\hline Sim & 674 & 326 & & \\
\hline Não & 137 & 113 & & \\
\hline Desconforto respiratório & & & $1,767(1,351-2,312)$ & 0,000 \\
\hline Sim & 544 & 255 & & \\
\hline Não & 169 & 140 & & \\
\hline Saturação de oxigênio <95\% & & & $2,115(1,621-2,761)$ & 0,000 \\
\hline Sim & 538 & 239 & & \\
\hline Não & 166 & 156 & & \\
\hline Vômito & & & $1,256(0,859-1,835)$ & 0,279 \\
\hline Sim & 89 & 51 & & \\
\hline Não & 360 & 259 & & \\
\hline Diarreia & & & $0,943(0,673-1,321)$ & 0,799 \\
\hline Sim & 106 & 76 & & \\
\hline Não & 352 & 238 & & \\
\hline
\end{tabular}

*Teste do qui-quadrado com correção de continuidade

Fonte: https://opendatasus.saude.gov.br/dataset/bd-srag-2020.

E quanto a faixa etária, cor da pele e doença hepática também alcançaram significância estatística $(p<0,05)$, havendo mais pessoas $\geq 65$ anos, com pele preta ou parda, e/ou com doenças hepáticas crônicas (fatores de risco) internadas nos hospitais públicos do que nos hospitais privados (Tabela 4.2). 
Tabela 4.2. Razões de Chances para fatores de risco entre pacientes internados em hospitais públicos em relação aos hospitais privados em 18 municípios da Região Metropolitana do estado do Rio de Janeiro entre março e novembro de 2020. Niterói, 2020.

\begin{tabular}{|c|c|c|c|c|}
\hline & $\begin{array}{c}\text { Hospitais } \\
\text { públicos (n) }\end{array}$ & $\begin{array}{c}\text { Hospitais } \\
\text { privados (n) }\end{array}$ & OR (IC 95\%) & P valor* \\
\hline \multicolumn{5}{|l|}{ Fatores de risco } \\
\hline Sexo & & & $1,190(0,975-1,452)$ & 0,096 \\
\hline Masculino & 565 & 297 & & \\
\hline Feminino & 502 & 314 & & \\
\hline Faixa etária & & & $1,386(1,135-1,693)$ & 0,002 \\
\hline$>=65$ anos & 554 & 268 & & \\
\hline$<65$ anos & 513 & 344 & & \\
\hline Cor da pele & & & $2,224(1,708-2,897)$ & 0,000 \\
\hline Preta ou parda & 423 & 152 & & \\
\hline Branca & 244 & 195 & & \\
\hline Doença cardiovascular crônica & & & $0,658(0,402-1,079)$ & 0,122 \\
\hline Sim & 376 & 238 & & \\
\hline Não & 60 & 25 & & \\
\hline Doença hematológica crônica & & & $1,513(0,461-4,964)$ & 0,687 \\
\hline Sim & 10 & 4 & & \\
\hline Não & 152 & 92 & & \\
\hline Síndrome de Down & & & $1,211(0,108-13,537)$ & 1,000 \\
\hline Sim & 2 & 1 & & \\
\hline Não & 152 & 92 & & \\
\hline Doença hepática crônica & & & $1,643(1,483-1,821)$ & 0,030 \\
\hline Sim & 10 & 0 & & \\
\hline Não & 143 & 92 & & \\
\hline Asma & & & $0,637(0,323-1,259)$ & 0,260 \\
\hline Sim & 20 & 19 & & \\
\hline Não & 147 & 89 & & \\
\hline Diabetes mellitus & & & $1,494(1,006-2,216)$ & 0,058 \\
\hline Sim & 262 & 115 & & \\
\hline Não & 90 & 59 & & \\
\hline Doença neurológica & & & $0,788(0,436-1,425)$ & 0,525 \\
\hline Sim & 33 & 24 & & \\
\hline Não & 143 & 82 & & \\
\hline Pneumopatia & & & $1,065(0,609-1,862)$ & 0,937 \\
\hline Sim & 48 & 25 & & \\
\hline Não & 137 & 76 & & \\
\hline Imunodepressão & & & $2,312(1,011-5,286)$ & 0,065 \\
\hline $\mathrm{Sim}$ & 29 & 8 & & \\
\hline Não & 138 & 88 & & \\
\hline Doença renal & & & $1,625(0,894-2,951)$ & 0,145 \\
\hline Sim & 47 & 19 & & \\
\hline Não & 134 & 88 & & \\
\hline Obesidade & & & $0,564(0,312-1,018)$ & 0,078 \\
\hline Sim & 27 & 29 & & \\
\hline Não & 137 & 83 & & \\
\hline
\end{tabular}

*Teste do qui-quadrado com correção de continuidade

Fonte: https://opendatasus.saude.gov.br/dataset/bd-srag-2020.

A chance de paciente de um hospital público ser internado em uma UTI foi 0,534 a chance em um hospital privado, ou seja, pouco mais da metade. Já a chance de óbito em hospitais públicos foi 3,334 vezes a chance de óbito em hospitais privados. As duas associações foram muito significativas (Tabela 5). 
Tabela 5. Razões de chances de internações em unidades de saúde hospitalares públicas e privadas entre aqueles que foram internados em UTI ou foram a óbito. Niterói,2020.

\begin{tabular}{|c|c|c|c|c|}
\hline & & & OR (IC95\%) & $\mathrm{P}$ valor** \\
\hline & \multicolumn{2}{|c|}{ UTI* } & $0,534(0,429-0,666)$ & $>0,001$ \\
\hline & $\operatorname{Sim}(n)$ & Não (n) & & \\
\hline Hospitais Públicos & 313 & 500 & & \\
\hline \multirow[t]{3}{*}{ Hospitais Privados } & 294 & 251 & & \\
\hline & \multicolumn{2}{|c|}{ Óbitos } & $3,334(2,543-4,473)$ & $>0,001$ \\
\hline & $\operatorname{Sim}(n)$ & Não (n) & & \\
\hline Hospitais Públicos & 346 & 721 & & \\
\hline Hospitais Privados & 77 & 535 & & \\
\hline
\end{tabular}

*UTI: Unidade de terapia intensiva

**Teste do qui-quadrado com correção de continuidade

Fonte: https://opendatasus.saude.gov.br/dataset/bd-srag-2020.

\section{Discussão}

A atenção hospitalar aos pacientes com SRAG, certamente a quase totalidade com COVID-19, foi dispersa. Os hospitais públicos atenderam a maioria, com maior concentração de pacientes com sinais ou sintomas associados a resultados desfavoráveis, e de pacientes com fatores de risco. A chance de um paciente ter passado por uma UTI em um hospital público foi pouco mais da metade do que em um hospital privado e a chance de morte foi cerca do triplo.

Esses resultados indicam que os pacientes internados nos hospitais públicos contaram com assistência da atenção primária falha uma vez que apresentavam mais sinais e sintomas apontando para um desfecho negativo, indicando uma demora para a internação.

Cabe ressaltar que em 2020, houve um aumento de 334\% no total de idosos mortos em domicílio, podendo estar relacionada também com uma falha no sistema de atendimento na saúde básica (Romero et al. 2020). Esses acontecimentos reforçam a conclusão de um estudo de 2017, o qual constatou que os municípios brasileiros com menor cobertura da atenção primária estão associados à uma maior mortalidade (Hone et al. 2017).

O Plano Nacional de Combate ao Coronavírus (Frente pela Vida) preconizou algumas medidas adicionais que o SUS deveria adotar para combater a pandemia. Dentre elas, a ampliação do acesso ao sistema - desde a atenção primária até redes hospitalares especializadas - uma qualificação profissional, expansão do horário de atendimento das unidades básicas de saúde (Frente pela Vida, 2020).

Entretanto, a variação no número de casos e mortes de COVID-19 refletem diferenças em fatores epidemiológicos e populacionais, bem como nas práticas clínicas e de saúde pública, que buscamos abordar nesse texto (Ribeiro et al. 2020). Como consequência da insuficiência de iniciativas na direção apontada, é possível observar desde o início da pandemia, a saturação do sistema de saúde público (Noronha et al., 2020). Pois, a partir de uma associação com a disponibilidade de leitos - públicos e privados - e a velocidade de propagação e infecção do vírus, foi estimado que um colapso no sistema de saúde brasileiro ocorreria quando a taxa de infecção fosse de, apenas, $1 \%$ da população (Noronha et al., 2020).

E ainda, houve considerável dispersão da assistência hospitalar (205 hospitais realizaram 1679 internações) o que fez com que até $25 \%$ do total de hospitais tenham internado somente um paciente com SRAG. Uma vez que incluímos somente casos residentes em 18 municípios da RM, não se sabe o total de internações realizadas por cada unidade hospitalar. No 
entanto, nas unidades fora da capital (74\% das internações), o número de internações de residentes na RM tende a se aproximar do total internado (Tabela 2).

Tal fato, nos coloca diante de um problema, onde a literatura mostra que uma das causas da diminuição da mortalidade por SRAG em 2020 foi a curva de aprendizado, como: experiência adquirida com as internações, uma melhor compreensão de como usar esteroides e uma mudança de drogas e procedimentos não comprovados (Ledford, 2020). Logo, os hospitais com menos internações tendem a levar mais tempo para consolidar essa experiência.

Ainda na Tabela 2, observa-se que em onze dos 18 municípios estudados 50\% ou mais das internações ocorreram em hospitais não sediados no município de residência do paciente. Além disso, hospitais públicos e privados do município do Rio de Janeiro hospitalizaram mais de $50 \%$ dos pacientes, em sete dos 18 municípios. A região da Baixada Fluminense (sub-região dentro da RM) conta com o Consórcio Intermunicipal de Saúde da Baixada Fluminense - CISBAF (https://cisbaf.org.br/). Entretanto, dentre os 745 casos internados por SRAG residentes na Baixada Fluminense, somente 391 (23\%) foram internados em unidades da Baixada. Portanto, essas informações transparecem a precariedade da rede de atendimento hospitalar na RM, revelando os problemas que o recrudescimento da pandemia pode implicar.

Os pesquisadores da área de saúde e da economia global recomendaram o isolamento social e a testagem do máximo de casos suspeitos para retomar o comércio, e quando houvesse diminuição no número de contaminados e mortes. Porém, isso não aconteceu em todos os países, como no Brasil, devido, também, ao grande número de trabalhadores informais e ao alto índice de desemprego já instaurado no país, que prejudicou o cumprimento das recomendações dos pesquisadores (Costa e Silva, 2020). E fica evidente que, o não cumprimento do isolamento por grande parte da população, a fragilidade de monitoramento, de testagem, de isolamento dos positivos e de acompanhamento dos doentes, traduz-se de forma a permitir oportuna hospitalização, visto que o Brasil é o $2^{\circ}$ com maior número de mortes e o $3^{\circ}$ com maior número de casos, apesar de ser o $6^{\circ}$ país em população (Johns Hopkins University, 2020; Worldometers, 2020).

Houve maior chance de indivíduos mais velhos, de cor da pele preta ou parda serem internados em hospitais públicos (tabela 4.2), bem como tem sido relatado que pacientes idosos sofrem mais complicações e têm prognóstico desfavorável (necessidade de internação em UTI de intervenção ventilatória invasiva e morte (Rincon et al., 2020). E o número de internações de indivíduos de cor da pele preta e parda revelam o maior estado de vulnerabilidade ao COVID-19. Pois, a falta de acesso aos hospitais (Oliveira et al., 2020), a busca tardia por atendimento e a alta exposição destas pessoas - devido ao fato de terem que trabalhar para garantir o sustento e ocuparem grande parte dos subempregos e atividades informais - leva a população preta e parda a chegar aos hospitais com casos graves da doença, onde há menor possibilidade de reversão do quadro (Faculdade de medicina UFMG 2020).

Quanto a idade, o risco de morte em pacientes com mais de 50 anos é 13\% maior, enquanto os pacientes com qualquer comorbidade têm risco de morte aumentado em até 39\% (Abate et al., 2020).

Neste estudo, mediante sintomas e fatores de risco, os indivíduos com dispneia, desconforto respiratório baixa saturação de $\mathrm{O}_{2}$, portadores de diabetes mellitus, doença hepática e imunossupressão também foram internados mais frequentemente em hospitais públicos. E todos esses sinais ou sintomas foram associados a piores desfechos.

Diante deste contexto, esses dados, aproximam-se de estudos como de uma coorte britânica de 5.693 pacientes com COVID-19 hospitalizados, onde o risco de morte foi mais comum em homens (HR 1,99, IC 95\% 1,88-2,10); idade avançada e diabetes não controlada (HR 2,36 IC 95\% 2,18-2,56 (Williamson et al., 2020). Além disso, na China, dados clínicos sobre 82 casos confirmados em laboratório como infecção por SARS-CoV-2 que foram a óbito em um hospital local de Wuhan mostram que a insuficiência respiratória foi a principal causa de morte $(69,5 \%$ ) (Zhang et al., 2020). E ainda, em uma série retrospectiva de casos em um único centro, dos 138 pacientes hospitalizados também em Wuhan, no período de $1^{\circ}$ de janeiro a 
28 de janeiro de 2020, pacientes que foram a óbito tiveram maior probabilidade de serem mais velhos, apresentar doenças subjacentes e apresentar sintomas de dispneia (Wang et al., 2020).

Ademais, um dos achados mais desconcertantes foi a ocorrência de baixos níveis de concentração de $\mathrm{O}_{2}$ em pacientes que não se queixavam de dispneia, o que se mostrou um desafio uma vez que a condição é preditora de piores resultados, daí a recomendação da mensuração regular do nível de $\mathrm{O}_{2}$ em casos suspeitos (Tobin et al., 2020). Outro estudo com pacientes com sintomas gástricos, também em Wuhan, foi encontrada a associação da presença de doença hepática crônica e gravidade da COVID-19 na admissão, com falência respiratória, choque e falência múltipla de órgãos com necessidade de ventilação mecânica e internação na unidade de terapia intensiva (UTI; 22,97\% versus 8,14\%; p<0,001) (Jin et al.,2020). Apesar da imunidade celular e humoral anormais serem as principais características de não sobreviventes com COVID-19 (Zhao et al., 2020), há evidências conflitantes. Pois, em uma revisão sobre o tema Cajamarca-Baron et al. (2020), concluiu-se que pacientes com câncer ou em tratamento recente do câncer e infecção por SARS-CoV-2 têm maior risco de resultados negativos. No entanto, em pacientes transplantados (renais, cardíacos e hepáticos), com patologias neurológicas (esclerose múltipla, neuromielite óptica, miastenia gravis), imunodeficiências primárias e infecção pelo vírus da imunodeficiência humana (HIV) em associação com imunossupressores, os estudos não mostraram tendência para resultados piores (Cajamarca-Baron et al., 2020).

Ainda nesta pesquisa, o fato dos hospitais públicos apresentarem uma chance de óbito de 3,334 a dos privados, nos parece estar relacionado tanto com a condição na qual os pacientes chegam às unidades quanto à falta de UTI, uma vez que a chance foi a metade nos hospitais públicos.

Um estudo realizado no Espírito Santo também observou-se que os pacientes dos hospitais públicos apresentavam maior número de comorbidades, como doenças cardiovasculares, renais e diabetes, enquanto em privados, a frequência desses pacientes era menor. (Maciel et al. 2020). No entanto, Garcia et al (2020), em um estudo com pacientes internados em Florianópolis, não encontrou tal diferença. Essa divergência pode ser explicada pelas diferentes características locais, tanto da assistência em saúde quanto populacional (Garcia et al. 2020).

\section{Conclusão}

O objetivo do presente estudo foi analisar dados do SIVEP-Gripe de 18 municípios da região metropolitana do Rio de Janeiro, de pacientes internados por SRAG em hospitais públicos e privados, sendo observado, de um modo geral, que os municípios estudados, exceto Niterói, não estão preparados, , para dar suporte aos seus residentes, principalmente, no cenário de aumento de número de casos de COVID-19.

Houve ainda discrepância tanto na capacidade de atendimento ao paciente crítico prestado pelos hospitais públicos e privados, com menos internações em UTIs nos públicos, e com maior risco de resultados desfavoráveis em unidades hospitalares públicas, o que possivelmente pode ter elevado o número de óbitos. Da mesma forma, constatou-se falhas na atenção básica especificamente na testagem dos contatos e no monitoramento dos casos de COVID-19, levando à demora na internação no momento oportuno.

\section{Referências}

Abate, S. M., Ahmed, A. S., Mantfardo, B. \& Basu, B. (2020). Rate of Intensive Care Unit admission and outcomes among patients with coronavirus: A systematic review and Metaanalysis. PLoS ONE, 15 (7), e0235653. https://doi.org/10.1371/journal.pone.0235653. 
Agencia Brasil (2020 2.) Covid-19 responde por 97,5\% dos casos de SRAG reportados no país. https://www.istoedinheiro.com.br/covid-19-responde-por-975dos-casos-de-srag-reportados-no-pais/, em 20/12/2020.

Agencia Brasil (2020). Novos dados, painel fornecem informações sobre a capacidade hospitalar da Covid-19. https://agenciabrasil.ebc.com.br/saude/noticia/2020-07/quatro-regioes-no-estado-do-rio-estao-em-risco-baixo-para-covid-19.

Cajamarca-Baron J., Guavita-Navarro D., Buitrago-Bohorquez J., Gallego-Cardona L., Navas A., Cubides H. et al. (2020). SARS-CoV-2 (COVID-19) in Patients with some Degree of Immunosuppression. Reumatologia Clinica, S1699-258X(20)30201-1. doi: 10.1016/j.reuma.2020.08.004. https://www.reumatologiaclinica.org/en-sars-cov-2-covid-19-in-patients-with-avance-S2173574320301295?newsletter=true\&coronavirus.

CISBAF (2020) Consórcio intermunicipal de saúde da baixada fluminense. https://cisbaf.org.br/.

Costa, B. L., \& Silva, M. A. (2020). Desigualdade para inconformados: dimensões e enfrentamentos das desigualdades no Brasil. Cegov.

Cruz, F. O. (2020). Monitoramento de casos de síndrome respiratória aguda grave notificados no SIVEP-Gripe. INFOGRIPE: http://info.gripe.fiocruz.br

G1 Portal de Notícias da Globo. RJ tem 24.473 mortes por coronavírus e mais de 406 mil casos confirmados. (2020). https://g1.globo.com/rj/rio-dejaneiro/noticia/2020/12/20/rj-tem-24473-mortes-por-coronavirus-e-mais-de-406-mil-casos-confirmados.html.

Garcia, P. L., Andrade, P. M., Pedebôs, A. L., Traebert, J., Traebert, A. S. E., Moura, V. G. (2020) Mortalidade por COVID-19 em hospitais públicos e privados de Florianópolis/SC. https://doi.org/10.1590/SciELOPreprints.1630 .

Goldberg, D (2020). Overwhelmed: hospitals engulfed by rebounding vírus. Recuperado em:https://www.politico.com/news/2020/10/16/pandemic-statesvirus-rebound-429753.

Governo do Estado do Rio de Janeiro. Secretaria de Saúde - Fichas Técnicas Municipais. (2020). Recuperado de Conexão Saúde. https://www.saude.rj.gov.br/informacao-sus/fichas-tecnicas-municipais.

Hone, T, Rasella, D, Barreto, M, Atun, R, Majeed, A, Millett, C. (2017) Large Reductions In Amenable Mortality Associated With Brazil's \& Primary Care Expansion And Strong Health Governance. Health Aff (Millwood), 36(1), 149-158. 10.1377/hlthaff.2016.0966. https://opendatasus.saude.gov.br/dataset/bdsrag-2020.

IBGE (2020) Instituto Brasileiro de Geografia e Estatística. Biblioteca. Estimativas da população residente no Brasil e Unidades da Federação com data de referência em $1^{\circ}$ de julho de 2020. https://biblioteca.ibge.gov.br/visualizacao/livros/liv101747.pdf.

IBGE. (2019) Instituto Brasileiro de Geografia e Estatística. Estimativas da população enviadas ao TCU. https://www.ibge.gov.br/estatisticas/sociais/populacao/9103-estimativas-de-populacao.html?=\&t=resultados.

IBM Corp. Released (2012). IBM SPSS Statistics for Windows, Version 21.0. Armonk, NY: IBM Corp.

IPEA (2020) Instituto de Pesquisa Econômica Aplicada. Plataforma IPEA.

Jin, X., Lian, J. S., Hu, J. H., Gao, J., Zheng, L., Zhang, Y. M., et al. (2020). Epidemiological, clinical and virological characteristics of 74 cases of coronavirus-infected disease 2019 (COVID-19) with gastrointestinal symptoms. Gut; 69(6),1002-9.

Johns Hopkins University of Medicine. (2020) Coronavirus Resource Center. COVID_19 Dashboard by the Center for Systems Science and Engineering (CSSE) at Johns Hopkins University (JHU). https://coronavirus.jhu.edu/map.html.

Karaca-Mandic, P. (2020) New data, dashboard provide information on Covid-19 hospital capacity. Statnews. https://www.statnews.com/2020/12/10/newdata-dashboard-provide-information-on-covid-19-hospital-capacity/.

Kawa, H., Correia, D. M. S., Silva, M. S. A., Lessa, M. P., Costa, S. P. O., Freitas, J. V., Gardengui, M. C. L., Andrade, et al. (2020) Padrões de ocorrência do COVID-19 nos municípios da Região Metropolitana do Rio de Janeiro e a vulnerabilidade social. Research, Society And Development, 9, e919108454, http://dx.doi.org/10.33448/rsd-v9i10.XX

Ledford, H. (2020). Why do COVID death rates seem to be falling? Hard-won experience, changing demographics and reduced strain on hospitals are all possibilities - but no one knows how long the change will last. Nature News. https://media.nature.com/original/magazine-assets/d41586-020-031324/d41586-020-03132-4.pdf

Maciel, L. E., Jabor, P., Júnior, G. E., Tristão-Sá, R., Lima, D. C. R., Reis-Santos, B., et al. (2020) Fatores associados ao óbito hospitalar por COVID-19 no Espírito Santo (2020). Epide miol. Serv. Saude Brasília, 29(4), e2020413, 202. 10.5123/S1679-49742020000400022

Ministério da Saúde (2015). Asis - Análise de Situação de Saúde / Ministério da Saúde, Universidade Federal de Goiás. - Brasília: Ministério da Saúde

Ministério da Saúde (2020 2) Secretaria de Vigilância em Saúde. SIVEP Gripe. Ficha de registro individual. Casos de Síndrome Respiratória Aguda Grave Hospitalizado. $\quad$ https://opendatasus.saude.gov.br/dataset/ae90fa8f-3e94-467e-a33f-94adbb66edf8/resource/54a46c6d-e0b5-40b7-8b7485450d22ace3/download/ficha-srag-final-27.07.2020_final.pdf.

Ministério da Saúde (2020 3). CNES. Cadastro Nacional de Estabelecimentos de Saúde. http://cnes.datasus.gov.br/.

Ministério da Saúde (2020) openDataSUS. SRAG 2020 - Banco de Dados de Síndrome Respiratória Aguda Grave - incluindo dados da COVID-19. https://opendatasus.saude.gov.br/dataset/bd-srag-2020. 
Research, Society and Development, v. 10, n. 1, e25710111611, 2021

(CC BY 4.0) | ISSN 2525-3409 | DOI: http://dx.doi.org/10.33448/rsd-v10i1.11611

Noronha, K. V. M. S., Guedes, G. R., Turra, C. M., Andrade, M. V, Botega. L, Nogueira, D., Calazans, J. A, Carvalho, L., Servo, L., \& Ferreira M. F. (2020). The COVID-19 pandemic in Brazil: analysis of supply and demand of hospital and ICU beds and mechanical ventilators under different scenarios. Cad Saude Publica, 17, 36(6), e00115320. https://doi.org/10.1590/0102-311x00115320.

Oliveira, R. G., Cunha, A. P., Gadelha, A. G. S., Carpio, C. G., Oliveira, R. B., \& Corrêa, R. M. (2020). Desigualdades raciais e a morte como horizonte: considerações sobre a COVID-19 e o racismo estrutural. Cadernos de Saúde Pública, 36(9), e00150120. http://dx.doi.org/10.1590/0102-311x00150120.

Rede. Projeto Governança Metropolitana no Brasil. https://opendatasus.saude.gov.br/dataset/bd-srag-2020/resource/54a46c6d-e0b5-40b7-8b74-85450d22ace3,

Ribeiro, H. V., Sunahara, A. S., Sutton, J., Perc, M., Hanley, Q. S. (2020). City size and the spreading of COVID-19 in Brazil. PLoS One, 15(9), e0239699. https://doi.org/10.1371/journal.pone.0239699.

Romero, D., Castanheira, D., Gracie, R., Rodrigues, J. M., Marques, A., \& Andrade, N. (2020). O excesso de óbitos de idosos no município do Rio de Janeiro analisado segundo o local de ocorrência. GISE/LIS/ICICT/Fiocruz, 2020. https://portal.fiocruz.br/documento/note-tecnica-o-excesso-de-obitos-de-idosos-nomunicipio-do-rio-de-janeiro-analisado.

SRAG 2020 - Banco de Dados de Síndrome Respiratória Aguda Grave - incluindo dados da COVID-19 - Open Data. (2020). Saude.gov.br

Tobin, M. J., Laghi, F., \& Jubran, A. (2020). Why COVID-19 Silent Hypoxemia Is Baffling to Physicians. American journal of respiratory and critical care medicine, 202(3), 356-360. https://doi.org/10.1164/rccm.202006-2157CP

UFMG, Faculdade de Medicina. (2020). População negra é mais vulnerável ao novo Coronavírus. Medicina UFMG: https://www.medicina.ufmg.br/populacao-negra-e-mais-vulneravel-ao-novo-coronavirus

Vida, F. P. (2020). Plano Nacional de Enfrentamento à pandemia da COVID-19. https://frentepelavida.org.br/uploads/documentos/PEP-COVID-19COMPLETO.pdf

Wang D., Hu B., Hu C., Zhu F., Liu X., Zhang J., et al.(2020) Clinical Characteristics of 138 Hospitalized Patients With 2019 Novel Coronavirus-Infected Pneumonia in Wuhan, China. JAMA; 323(11):1061-9. https://doi.org/10.1001/jama.2020.1585 pmid:32031570

Wang, J., Lee, Y., Liu, F., \& Zhou, M. (2020). To relax restrictions: Are communities ready to deal with repeated epidemic waves of COVID-19? Infection Control \& Hospital Epidemiology, 1-2. 10.1017/ice.2020.228

Williamson, E., Walker, A. J., Bhaskaran, K. (2020) Factors associated with COVID-19-related hospital death in the linked electronic health records of 17 million adult NHS patients. J Chem Inf Model;53:1689-1699. https://www.nature.com/articles/s41586-020-2521-4.

Worldometers (2020). https://www.worldometers.info/coronavirus/.

Zhang, B., Zhou, X., Qiu, Y., Song, Y., Feng, F., Feng, J., et al. (2020) Clinical characteristics of 82 cases of death from COVID-19. PLoS ONE 15(7): e0235458. https://doi.org/10.1371/journal.pone.0235458

Zhao, Y., Nie, H. X., Hu, K., Wu, X. J., Zhang, Y. T., Wang, M. M. et al. (2020). Abnormal immunity of non-survivors with COVID-19: predictors for mortality. Infect Dis Poverty.Aug 3;9(1):108. 10.1186/s40249-020-00723-1 